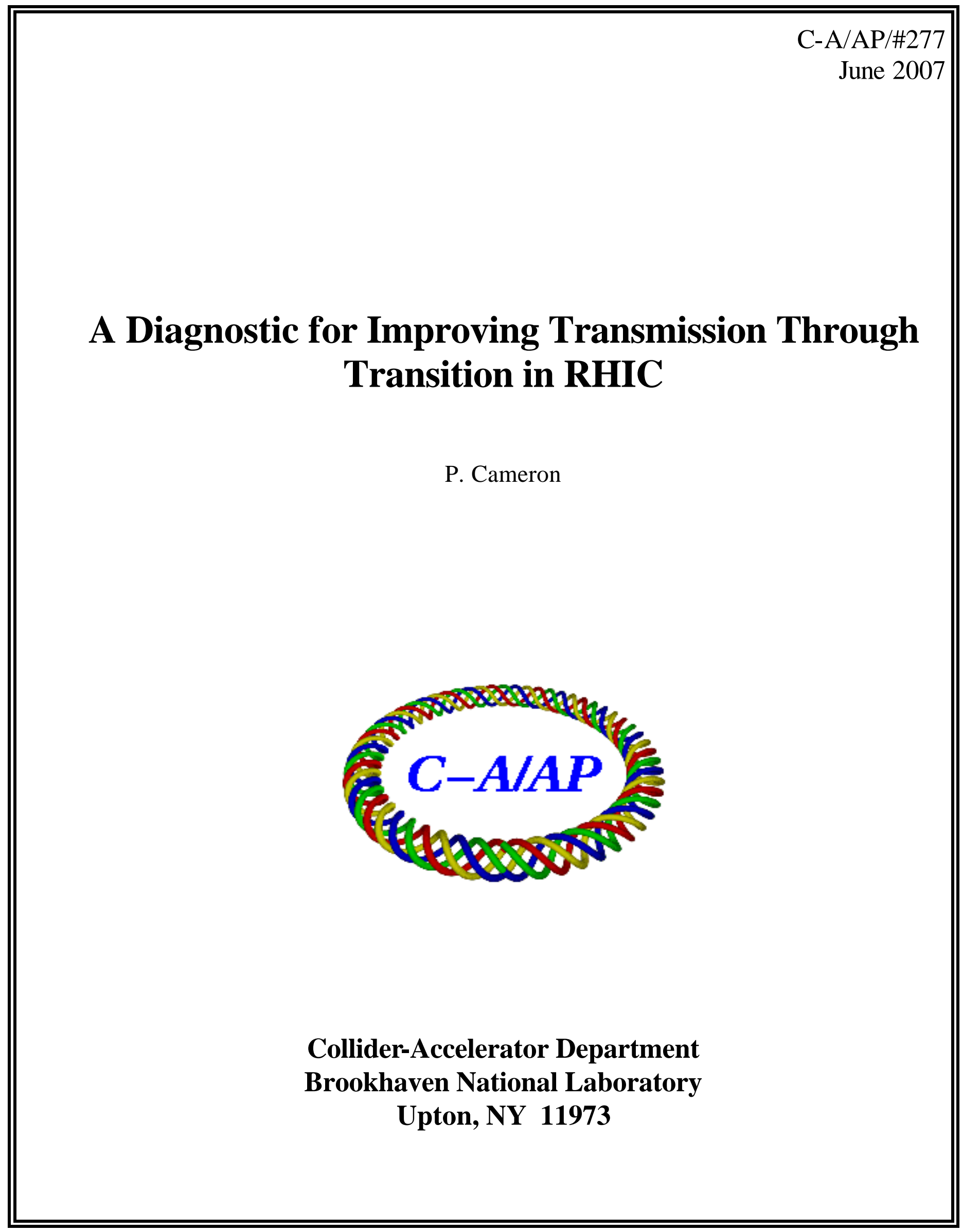




\title{
A DIAGNOSTIC FOR IMPROVING TRANSMISSION THROUGH TRANSITION IN RHIC
}

\author{
Peter Cameron, C-AD, BNL, Upton, NY 11973, USA
}

\section{INTRODUCTION}

For species other than protons, RHIC luminosity is limited by emittance growth at transition. The present understanding $[1,2]$ is that this results from a fast single bunch instability, promoted by the presence of electron cloud. The character is similar to the head-tail instability or, in linacs, to the beam break-up instability. Possible cures to this instability include scrubbing to reduce pressure rise and the consequent electron cloud, a broadband damper (with bandwidth approaching the $\mathrm{GHz}$ range) to damp the instability, and improved chromaticity control. This note addresses the possibility of implementing a diagnostic for high time resolution ( $<100 \mathrm{msec})$ chromaticity measurement in the vicinity of transition, using immediately available hardware.

Using the radial modulation method, the baseband tune tracker delivers useful chromaticity information on the acceleration ramp (although the quality is compromised by mains harmonics $[3,4]$ ), excepting the time from shortly before transition until a few seconds after transition. Data is absent at precisely the time when it is most needed for optimizing transition crossing. The following sections outline an approach to obtaining the needed data. It would be particularly useful to explore this method during the present RHIC run, so that results can be evaluated and improvements can be made for the 2008 run.

\section{PRELIMINARY RESULTS AND PROPOSED SYSTEM ARCHITECTURE}

Preliminary results date back to the very first successful transition crossing in RHIC. Figure 1 shows the FFT of the signal from the $2 \mathrm{GHz}$ resonant Schottky pickup during that transition crossing, which occurred during the RHIC 2000 run.

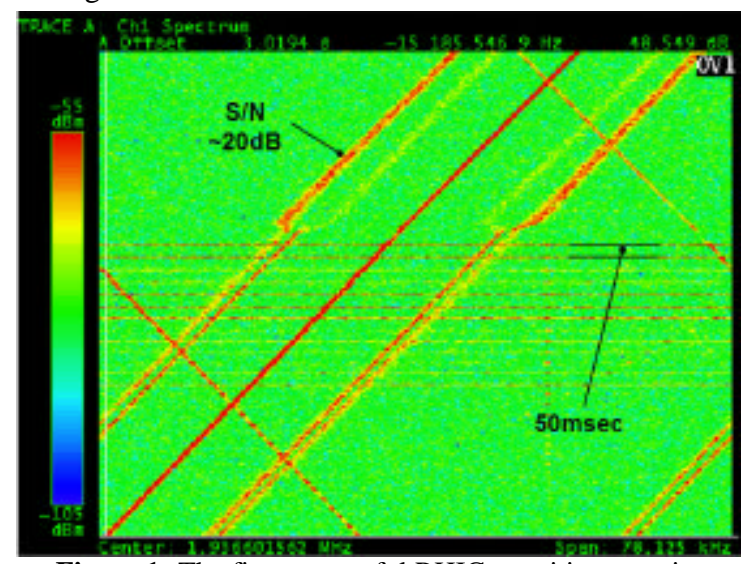

Figure 1: The first successful RHIC transition crossing
In this image the most recent time is at the bottom of the figure. The frequency slewing results from the fact that a synchronous local oscillator was not available for mixing down the Schottky signal. The sidebands from both betatron eigenmodes are visible, as a result of coupling. At that time transition crossing was accomplished by the radial jump method, and the magnitude and sign of the chromaticity could be determined from the clearly visible tune jump. The periodic broadband noise after transition resulted from beam loss in dispersive regions due to longitudinal quadrupole oscillations. Even with the low intensity available then, it is clear that useful information was available in the Schottky spectrum.

In the present operational configuration, extracting chromaticity information from Schottky signals is complicated by two operational improvements. First, the ramp rate is greatly increased, requiring a synchronous local oscillator to remove the frequency slewing. And second, transition crossing is now accomplished by fast quadrupoles rather than the radial shift, so that chromaticity information must be extracted from the relative widths of the betatron sidebands, rather than the tune jump at transition. While this complicates the measurement, it has the advantage that chromaticity information can be made available continuously in the vicinity of transition, rather than just at the jump.

Presently, with gold ions in RHIC the signal-to-nois e ratio of the $2 \mathrm{GHz}$ Schottky system at store it is typically $\sim 30 \mathrm{~dB}$ at the betatron lines with $\sim 128 \mathrm{~Hz}$ resolution bandwidth. Around transition the Schottky betatron linewidths are narrow (by definition the revolution line width vanishes at transition), and he spectral power density at the betatron lines is much greater than at store. This suggests that in the vicinity of transition it might be possible to obtain clean spectra (signal-to-noise ratio of $40 \mathrm{~dB}$ or better) with good time resolution (on the order of $100 \mathrm{msec}$ or less). This has been confirmed by recent preliminary measurements.

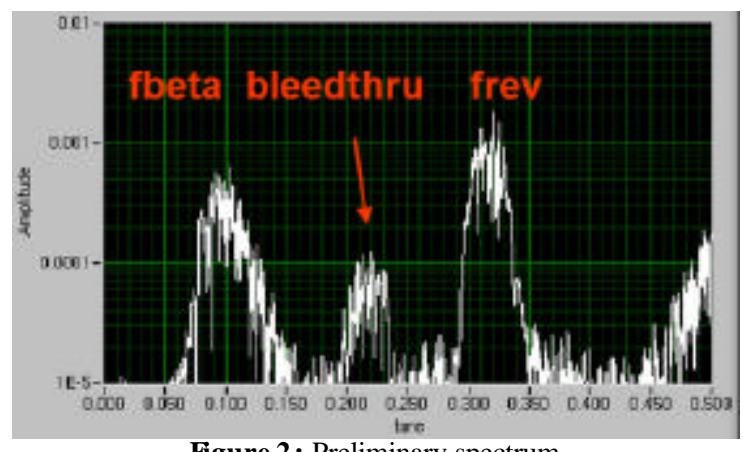

Figure 2: Preliminary spectrum 
Figure 2 shows the FFT of $26 \mathrm{msec}$ of data taken on the horizontal plane of the blue ring shortly before transition, using the Beam Transition Monitor data acquisition system. The frequency span of this monitor is limited by the digitizer clock frequency to $\sim$ half the revolution frequency, resulting in truncation of the upper betatron line. The revolution line and lower betatron line are clearly visible, as well as the revolution line bleeding through from the orthogonal cavity mode. The data acquisition and signal processing for this measurement was not optimized. It is estimated [5] that an improvement of $>15 \mathrm{~dB}$ in signal-to-noise is possible with optimized data acquisition, and further improvement is possible with attention to signal processing [6].

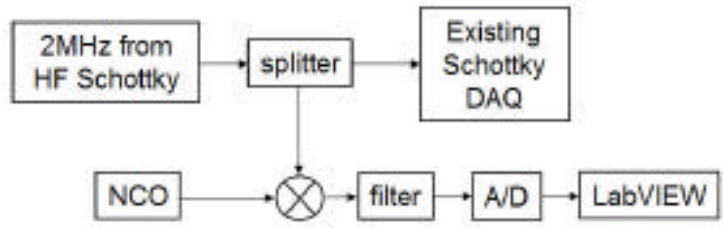

Figure 3: Proposed system block diagram

A system block diagram for the proposed diagnostic is shown in Figure 3. The NCO generates the synchronous IO needed to eliminate the frequency slewing. Chromaticity is extracted from the FFTs in LabVIEW.

\section{LINEWIDTH BEHAVIOR IN VARIOUS TRANSITION SCENARIOS}

Information about chromaticity is contained in the relative linewidths of the upper and lower betatron
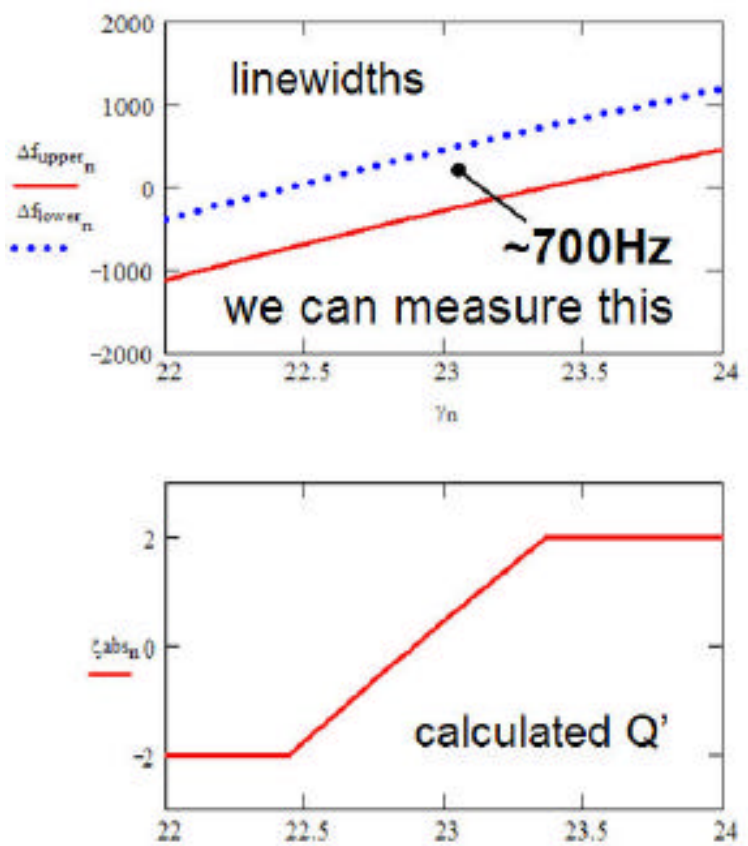

sidebands. At injection or store the chromaticity is calculated by simply multiplying the difference in these linewidths by a numerical factor. In the vicinity of transition the situation is more complicated.

This section presents linewidths in four different transition scenarios. In the first, chromaticity is constant in the vicinity of transition. In the second chromaticity is negative before transition and positive after, and jumps 'instantaneously' at transition. The third and fourth show the linewidth behaviour with a quadrupole jump at transition, the third with a chromaticity jump of 4 units, and the fourth with an approximation to the 'design + trim' chromaticities.

The $3 \mathrm{~dB}$ betatron linewidths are [7]

$$
\begin{aligned}
& \Delta f_{\text {upper }}=2 \sqrt{\ln 2} \cdot \frac{\delta p}{p} \cdot f_{\text {rev }} \cdot\left((h-Q) \cdot \eta-Q^{\prime}\right) \\
& \Delta f_{\text {lower }}=2 \sqrt{\ln 2} \cdot \frac{\delta p}{p} \cdot f_{\text {rev }} \cdot\left((h+Q) \cdot \eta+Q^{\prime}\right)
\end{aligned}
$$

where

$$
\begin{aligned}
& \frac{\delta p}{p}=\text { rms momentum spread } \\
& f_{\text {rev }}=\text { revolution frequency } \approx 78122 \mathrm{~Hz} \\
& h=\text { harmonic number } \approx 26000 \\
& Q=\text { integer plus fractional tune } \\
& Q^{\prime}=\text { chromatictiy } \\
& \eta=\text { relativisitc slip factor }=\frac{1}{\gamma^{2}}-\frac{1}{\gamma_{t}^{2}}
\end{aligned}
$$
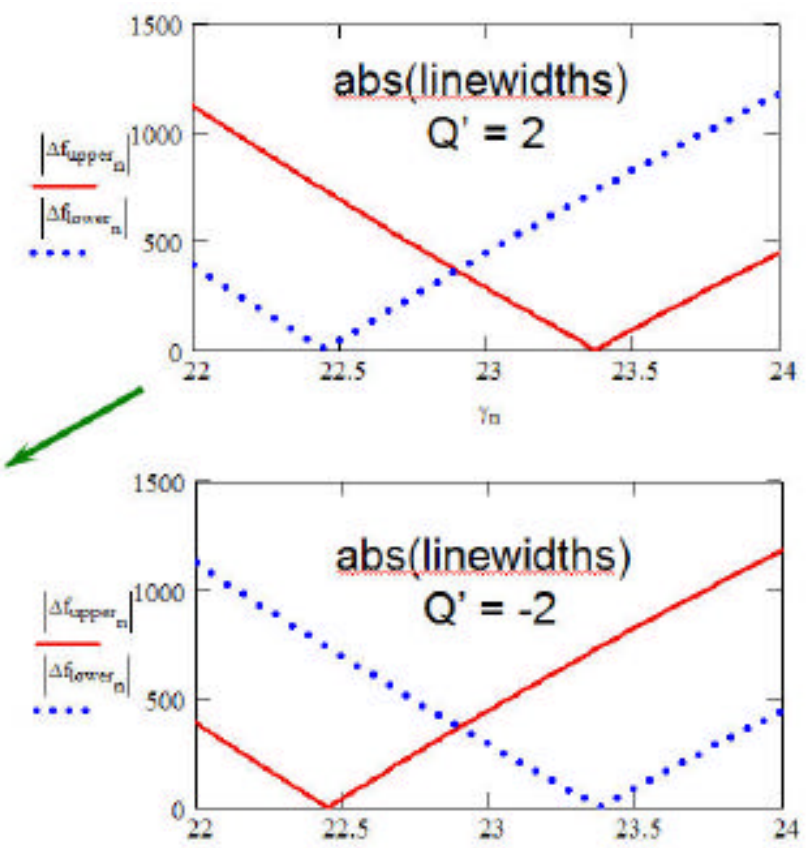

Figure 4. Linewidths and calculated chromaticity for Case 1 (chromaticity constant through transition) 


\section{Case 1. Constant chromaticity}

Figure 4 shows linewidths and chromaticities calculated from equations (1) for an rms momentum spread of .001, with no tune jump and constant chromaticity through transition, and assuming transition at $\gamma_{\mathrm{t}}=22.9$. With the exception of the lower right pane in this figure, the chromaticity $Q^{\prime}=2$. For comparison, in the lower right pane chromaticity is taken to be negative, rather than positive.

An obvious feature of upper left paneis the presence of 'negative' linewidths. Negative linewidths are not physically meaningful. The upper right and lower right panes show the absolute values of the linewidths for constant chromaticities of 2 and -2 . These are the physical 'observables'. It should be noted that information is lost by taking the absolute values of the linewidths.

Whereas the revolution linewidth goes to zero at transition, the upper and lower betatron linewidths go to zero (the octupoles at $\gamma_{\mathrm{t}}$ will broaden these lines by $\sim 1 \mathrm{KHz})$ on opposite sides of transition The distance from $\gamma_{t}$ at which the linewidths go to zero is proportional to the chromaticity.

The linewidth expressions presented above can be solved for chromaticity. The result must be expressed in terms of the physically observable linewidths.
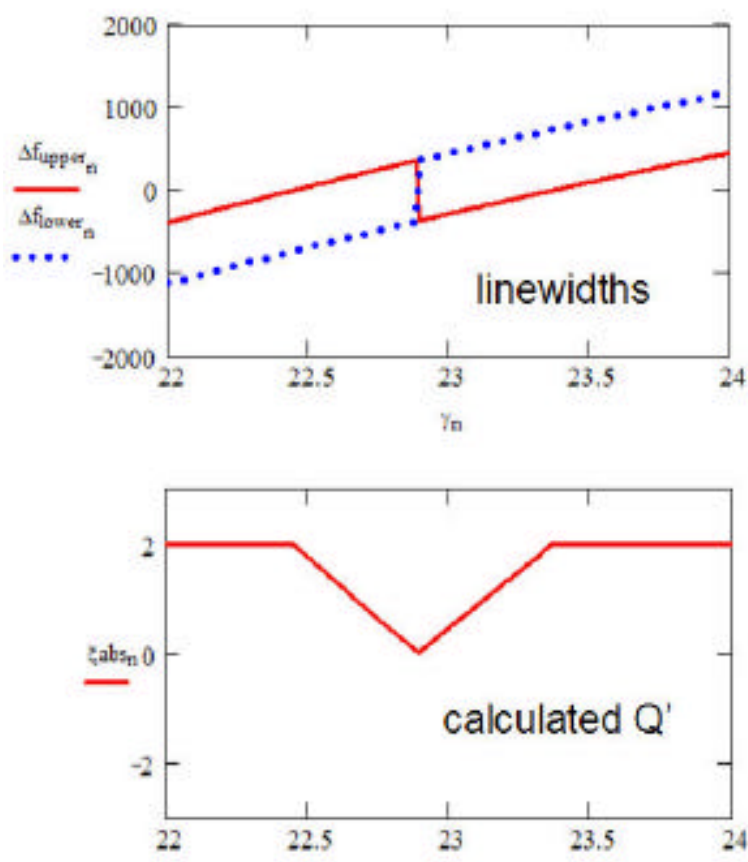

Figure 5. Linewidths and calculated chromaticity for Case 2 (chromaticity jump at transition)

\section{Case 2. Chromaticity jump at transition}

Figure 5 shows linewidths and chromaticities calculated for an rms momentum spread of .001, with chromaticity Q' equal to -2 before transition and +2

$$
Q^{\prime}=\frac{\left|\Delta f_{\text {lower }}\right|-\left|\Delta f_{\text {upper }}\right|}{4 \cdot \sqrt{\ln 2} \cdot \frac{\delta p}{p} \cdot f_{\text {rev }}}-Q \cdot \eta
$$

The lower left pane shows chromaticity calculated using this expression, for the linewidths of the upper right pane. It is obvious that the usual method of calculating chromaticity from measured linewidths is not well behaved around transition. The resulting calculated chromaticity is piecewise continuous. How to formulate and implement an algorithm to correctly calculate chromaticity in the various segments is not obvious. The situation is complicated by the loss of the sign information of the linewidths (negative linewidths are not observable), as well as by the tune and chromaticity jumps (see the following sub-sections). This is particularly true if one is confronted with the circumstance of multiple zero crossings of the chromaticity, as may presently be the circumstance in RHIC (see the 'Case 4' sub-section). However, for the purpose of tuning RHIC chromaticity around transition, it may not be essential to have this algorithm. It may be sufficient to simply observe the evolution of the measured linewidths (presuming an instrument is available to permit this observation), and by comparison with examples of the type presented in the following subsections, to make the deductions needed to accomplish the tuning.
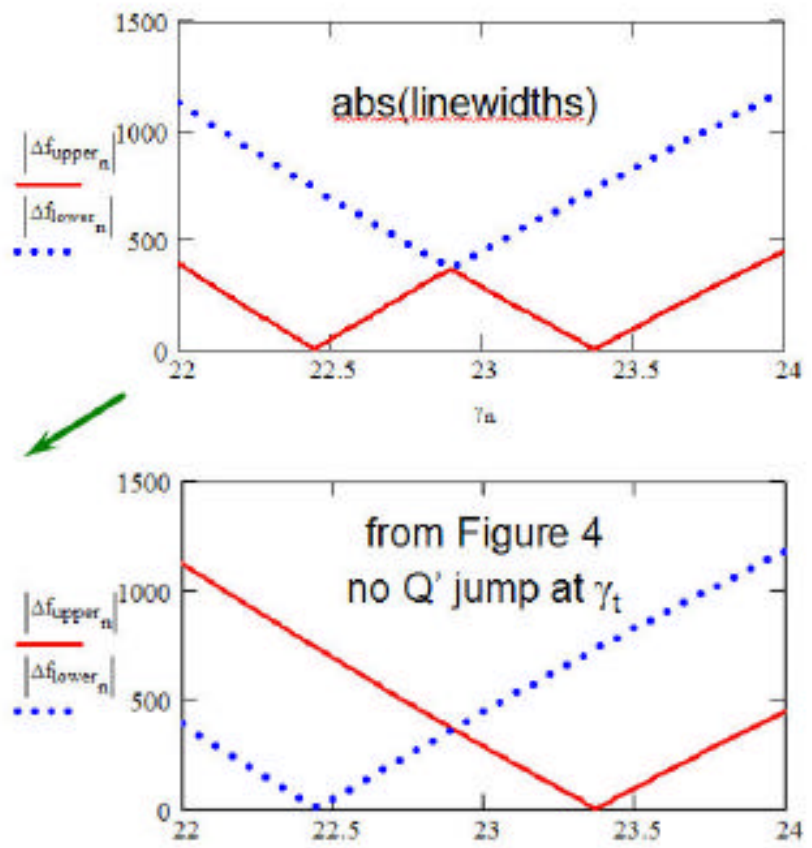
calculated chromaticity based on the observed values of the linewidths. For purpose of comparison, the lower right pane shows observed linewidths with no chromaticity jump.

In the previous sub-section it was shown that, with no chromaticity jump at transition, the upper and lower betatron linewidths go to zero on opposite sides of transition. With a chromaticity jump at transition the shrinkage of the lower sideband is interrupted before it approaches its theoretical minimum width and the upper sideband has minima on both sides of transition .
The calculated chromaticity looks significantly different from the input 4 unit jump at transition.

\section{Case 3. Tune and simple chromaticity jump at transition}

The fast transition quads cause a downwards jump in $\gamma_{t}$ of about one unit. This is shown in Figure 6 for the yellow ring. Here it is again assumed that Q' is equal to -2 before transition and +2 after transition. Including the effect of the $\gamma_{\mathrm{t}}$ jump, the appearance of Figure 5 of the previous sub-section is modified as shown in Figure 7 below.

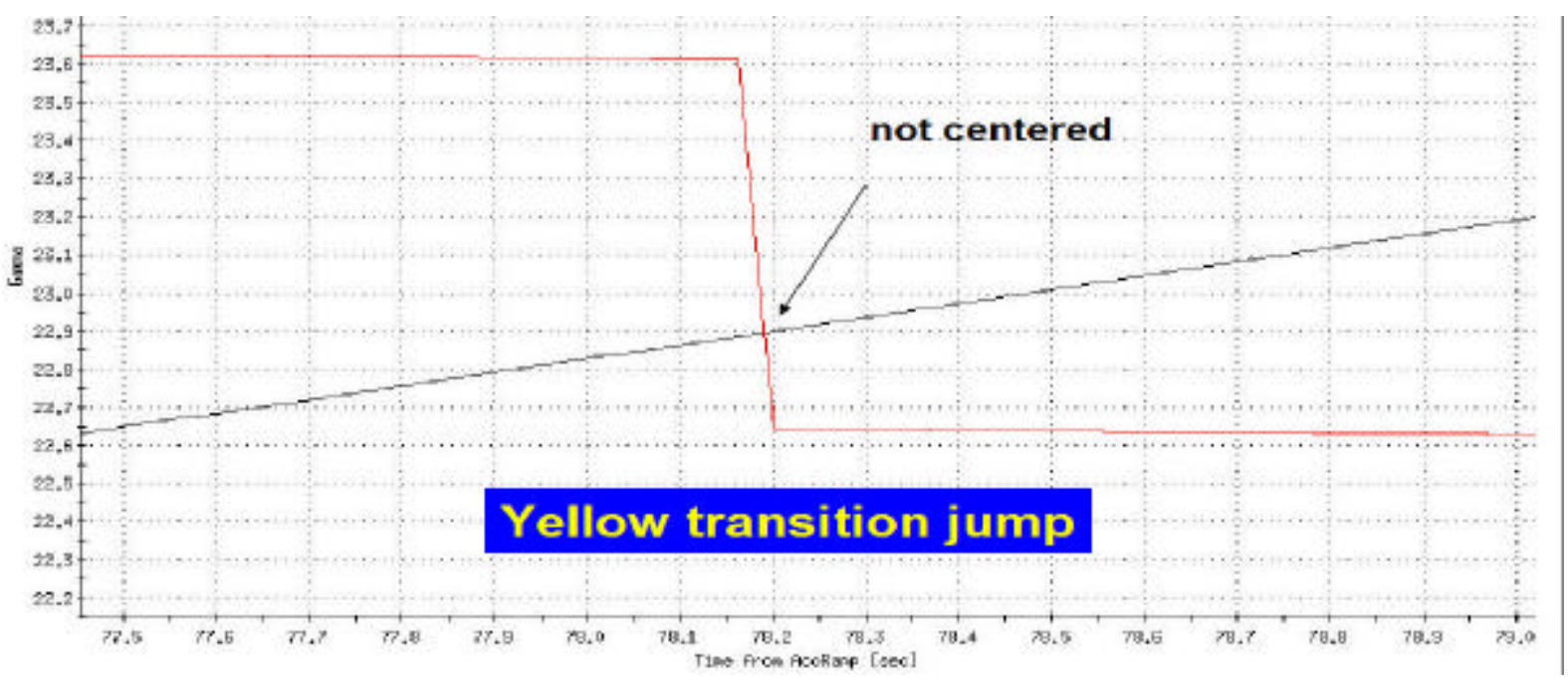

Figure 6: Transition jump in yellow

The shrinkage of the lower sideband is interrupted by the jump before it approaches its theoretical minimum width. The upper sideband is only
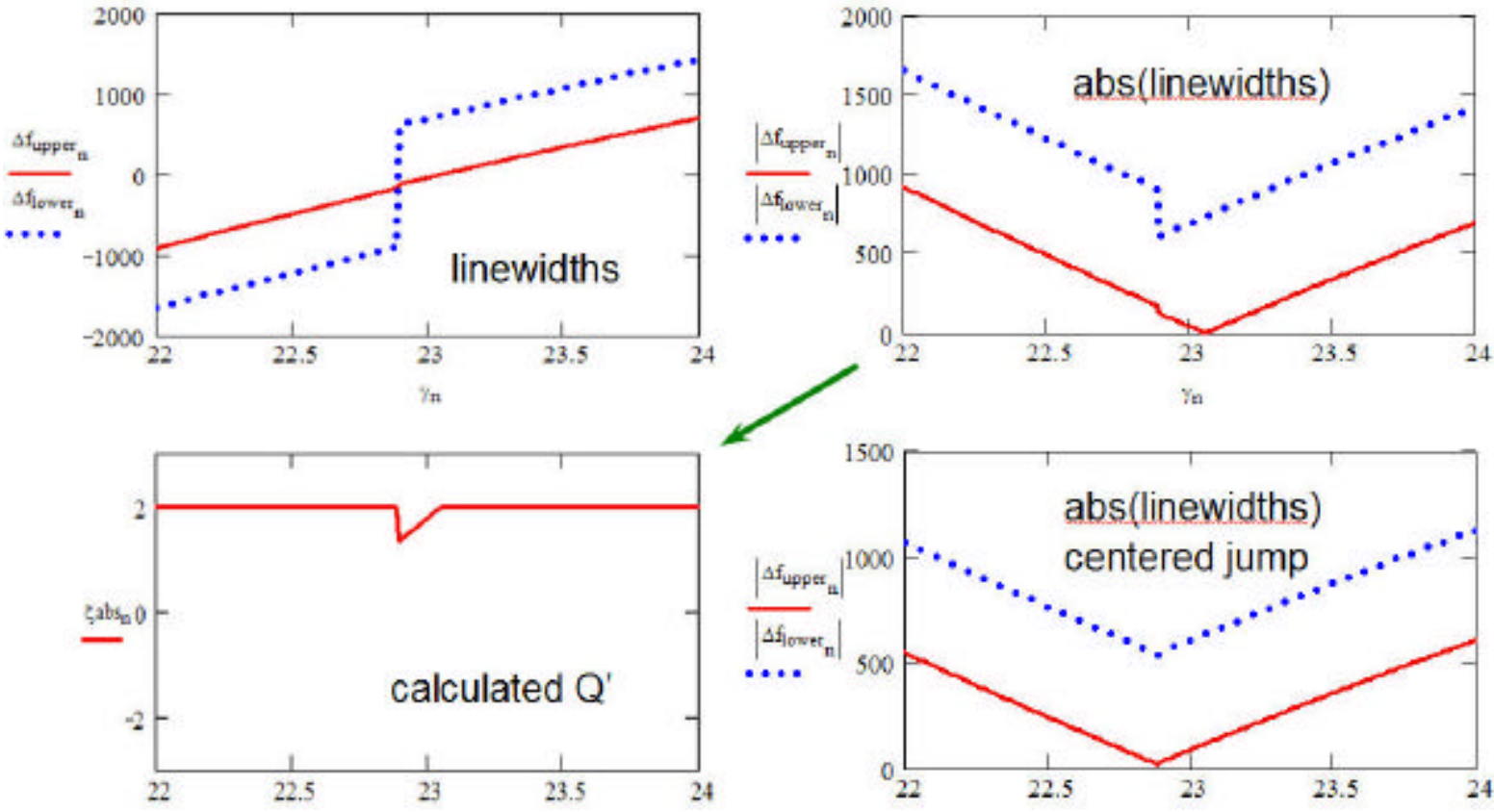

Figure 7: Linewidths and calculated chromaticity for Case 3 (tune and simple chromaticity jump at transition) 


\section{Case 4. Tune and chromaticity jump with 'design + trim' values for chromaticity}

The yellow ring ramp 8719 'design' and 'trim' values for chromaticity in the vicinity of transition are shown in Figure 8. A simple approximation of the 'design + trim' value of horizontal chromaticity is also shown in the figure, and was used to gener ate the results shown in Figure 9.

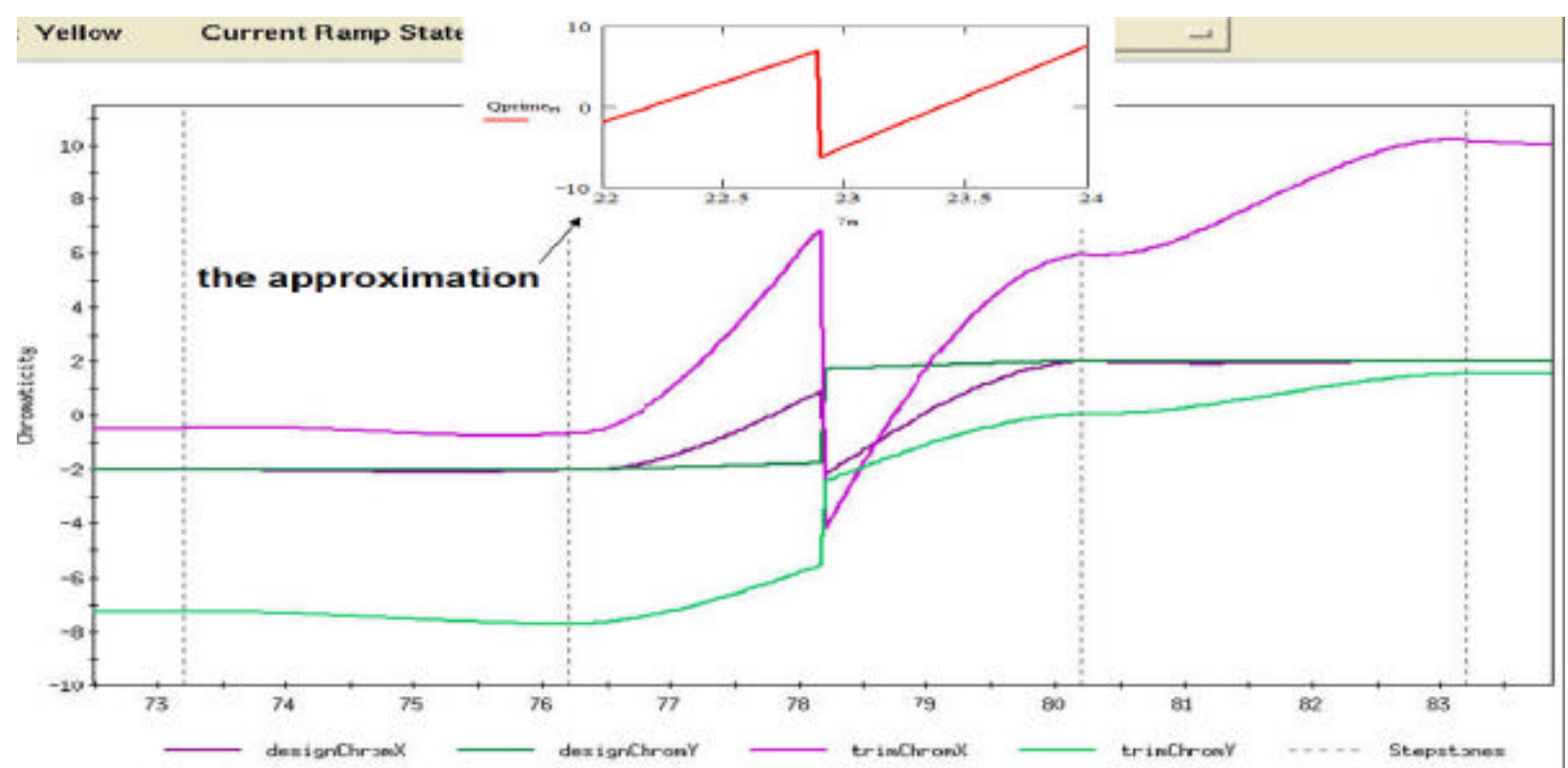

Figure 8: Yellow ring design and trim chromaticities around transition, and the approximation (inset) used to calculate the results shown in Figure 9

Figure 9 is a bit of a mess. It is not obvious how one would look at the 'observable' linewidths in the upper right pane, and from this extrapolate back to the lower right pane, which is the chromaticity input to the algorithm that calculates the linewidths. Comparison of the lower left and right panes is at least a little disturbing. The difference in appearance between the input and calculated chromaticities is remarkable.
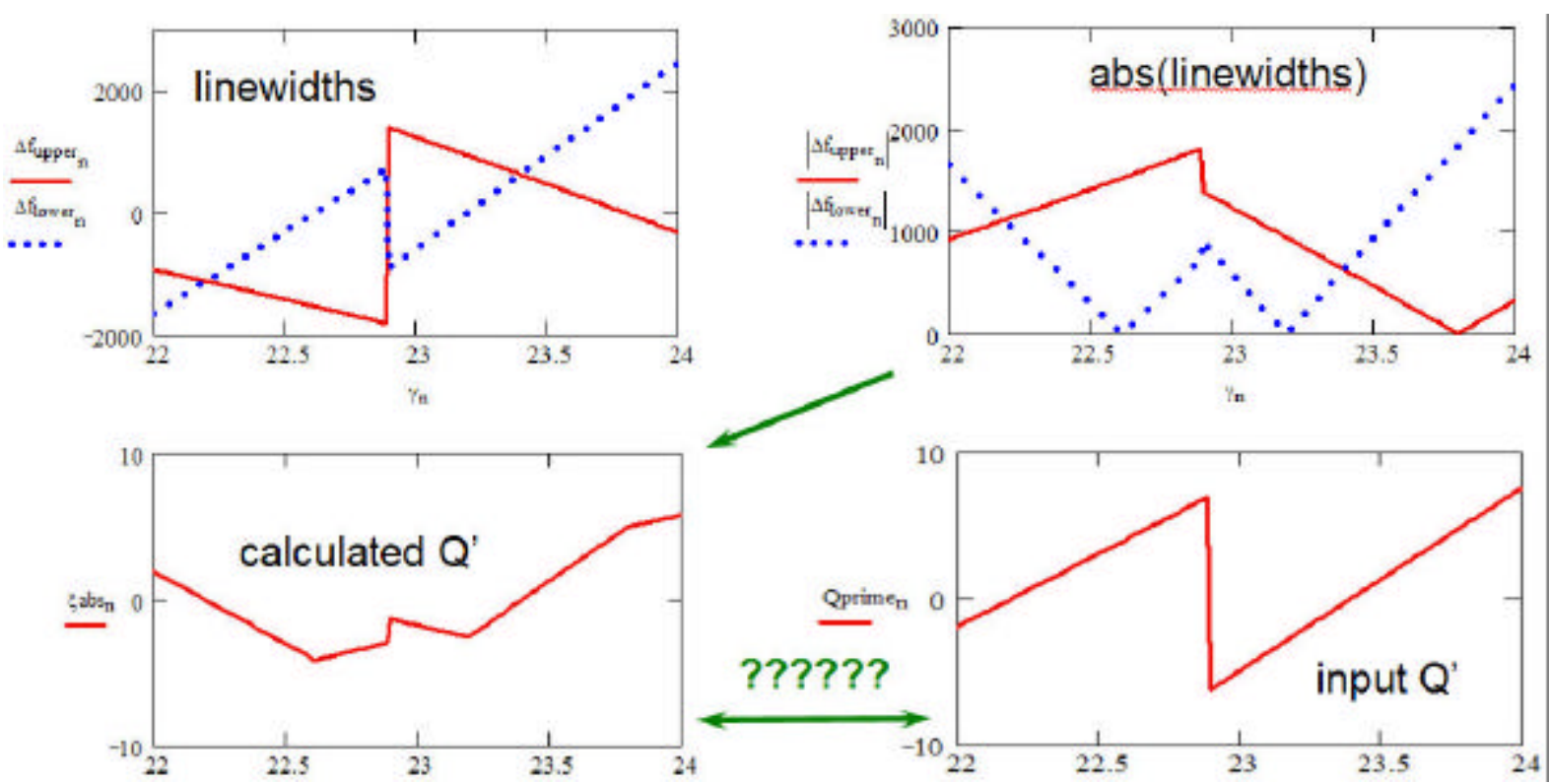

Figure 9: Linewidths and calculated chromaticity for Case 4 (tune and Figure 8 chromaticity jump at transition) 


\section{SUMMARY}

The behaviour of the betatron linewidths at transition is complicated by the fact that their minima are offset from the narrowing of the revolution line by chromaticity, as well as by the fact that the $\gamma_{t}$ jump introduces a discontinuity in this behaviour. This behaviour has been modelled for four scenarios of progressively increasing complexity, the last being an approximation to the present RHIC 'design + trim' chromaticities. The interpretation of this behaviour is further complicated by the loss of information that results from the impossibility of observing 'negative' frequencies.

Just the same, it should not be too difficult to learn how to extract the needed chromaticity from the Schottky signal. The signal has good signalto-noise. Interpreting the evolution of the spectrum through transition is simplified by the fact that the starting point will be known reasonably well. And the model presented here for calculating the observed linewidths is reasonably simple and straightforward, and can be fit to measurement results without undue difficulty.

\section{CONCLUSION}

For species other than protons, RHIC luminosity is limited by emittance growth at transition. Independent of the other possible approaches (scrubbing, broadband damper,...) to improving this situation, it would be useful to have a diagnostic that delivers chromaticity information around transition with good accuracy and time resolution. Without this the tuning effort is reduced to an inefficient process that closely resembles guessing.

At the present time the oly evident diagnostic possibility is the approach outlined in this note. Implementation of this approach may not be completely trivial, but, unless another method can be found to get chromaticity information around transition, it would be helpful to gain some experience with this measurement technique, preferably as soon as possible.

\section{REFERENCES}

[1] M. Blaskiewicz, private communication

[2] W. Fischer, private communication

[3] P. Cameron et al, "The Effects and Possible Origins of Mains Ripple in the Vicinity of the Betatron Spectrum", DIPAC 2005, Lyon.

[4] P. Cameron et al, "Observations of Direct Excitation of the Betatron Spectrum by Mains Harmonics in RHIC", C-AD AP Note 253.

[5] P. Cameron, presentation to the RHIC Weekly Monday Meeting, 14 May 2007.

[6] M. Gasior, "Improving the Frequency Resolution of Discrete Spectra", $\mathrm{PhD}$ thesis, available at

http://mgasior.web.cern.ch/mgasior/pap/gasior_phd.pdf [7] S. Chattopadhyay, "Some fundamental aspects of fluctuations and coherence in charged-particle beams in storage rings", CERN SPS 8411, available at http://documents.cern.ch/cgibin/setlink?base $=$ cernrep\&categ $=$ Yellow Report\&id=1 984-011 